\title{
Efecto de la hipoterapia en áreas cognitivas y psicomotoras en paciente con retraso psicomotor
}

\section{Effect of hyppotherapy on cognitive and psycomotor areas in psychomotor-retarded patients}

\author{
Carlos Alfonso Fierro Aldana ${ }^{1}$, Eduardo Tula Garzón ${ }^{1}$, Víctor Javier Vera Cárdenas ${ }^{2}$ \\ 1 Esp. en gerencia de la Salud Ocupacional, Docente Tiempo Completo Programa de Psicología, \\ Universidad Cooperativa de Colombia. \\ 2 Esp. Gestión del Desarrollo Humano y Bienestar Social, Docente Tiempo Completo Programa de \\ Psicología, Universidad Cooperativa de Colombia. \\ Correspondencia: Carlos Alfonso Fierro Aldana, Correo electrónico: carlos.fierro@campusucc. \\ edu.co
}

Fierro C, Garzón E, Vera V, Effect of hyppotherapy on cognitive and psychomotor areas in psychomotor-retarded patients. RFS - Revista Facultad de Salud. 2013; 5 (2): 70-78

\section{Resumen}

Introducción: Para demostrar el efecto de la hipoterapia en áreas cognitivas y psicomotoras en un paciente con retraso psicomotor que asiste a la Fundación Centro de Equinoterapia IPS se realizó un estudio controlado no aleatorio de tipo cuasi-experimental, con mediciones pre y postest. La paciente fue una menor con edad cronológica de 7 años 9 meses con diagnóstico de retraso psicomotor.

Materiales y Métodos: Se emplearon dos pruebas neuropsicológicas, el Cuestionario Cumanín (madurez neuropsicológica infantil), y la Escala McCarthy de aptitudes y psicomotricidad. Como variable independiente (VI) se consideró el tratamiento, mientras que la variable dependiente (VD) fueron las áreas cognitivas y psicomotoras.

Resultados: Las puntuaciones totales en desarrollo verbal fueron 10 en el pretest y 35 en el postest, mientras que el lenguaje no verbal puntuó 30 en el pre y 45 en el postest.

La conversión de la puntuación directa en desarrollo global a cociente de desarrollo (CD) indicó una puntuación de 82 en el pre y 95 en el postest.

Conclusiones: La aplicación del procedimiento hipoterapéutico (VI) mejoró el índice de desarrollo del paciente a nivel de psicomotricidad, lenguaje, estructuración espacial, visopercepción, no se modificaron las áreas de lenguaje articulatorio, atención y escritura, sin embargo, se presentó un decremento en la memoria icónica, (registro de la memoria sensorial relacionada con el dominio visual). 
Palabras clave: Terapia ecuestre, hipoterapia, psicomotricidad, retraso psicomotor.

\begin{abstract}
Introduction: to show the effect of hippotherapy on cognitive and psychomotor areas in a retarded patient attending Fundación Centro de Equinoterapia IPS, it was conducted a quasi-experimental non-randomized controlled study, with pre and post-test measurements. The patient was a female minor, seven years, nine months old, with a psychomotor retardation diagnosis.

Materials and methods: Two neuropsychological tests were used, the Cumanin Questionnaire (child neuropsychological maturity), and McCarthy Scale of aptitudes and psychomotor. Treatment was the independent variable (IV) while cognitive and psychomotor areas were the dependent variables (DV)

Results: Total score in verbal development was 10 in the pre and 35 in the post-test, while non-verbal language scored 30 in pre and 45 in post-test. Direct score conversion in overall development to development quotient (DQ) showed a 83 score in pre and 95 in post-test.

Conclusions: The application of hippotherapeutic procedure improved the patient's development index at psychomotor, language, spatial structuring and visual perception level. Articulatory language, attention and writing areas were not modified; nevertheless, there was a decrease in iconic memory (sensory memory record related to visual domain).
\end{abstract}

Key words: equine therapy, hippotherapy, psychomotor, psychomotor retardation.

\section{Introducción}

Las terapias ecuestres en la actualidad son ampliamente practicadas $^{(1)}$ en éstas se utilizan equinos (Equusferuscaballus) como instrumento terapéutico, se divide en tres áreas: Hipoterapia, monta terapéutica y volting $\mathrm{y}$, equitación como deporte para discapacitados.

Cada área está dirigida a diferentes tipos de discapacidad utilizando estrategias terapéuticas distintas. La terapia ecuestre integra cuatro ámbitos profesionales diferentes: la medicina, la psicología, la pedagogía y el deporte. El área médica desempeña un papel dominante en la hipoterapia porque la monta a caballo se utiliza a manera de fisioterapia, indicada para pacientes con disfunciones neuromotoras de origen neurológico, traumático o degenerativo ${ }^{(2)}$.

La psicología y pedagogía adquieren más importancia en la monta terapéutica, ya que la monta a caballo, además de cumplir su papel de fisioterapia, trata disfunciones psicomotoras, sensomotoras y sociomotoras funcionando así como una psicoterapia.

En la equitación como deporte para discapacitados la monta a caballo se realiza como deporte; esto permite que personas con cierta discapacidad física y funcional se integren a la vida deportiva.

En la monta terapéutica se une el objetivo terapéutico a la enseñanza específica de la equitación como deporte. Además de la realización de ejercicios neuromusculares y gimnásticos en combinación con juegos terapéuticos, el paciente aprende a utilizar las señales específicas de la equitación (ayudas de montar) para convertirse en un jinete activo. El paciente mueve su caballo solo en la pista y monta con silla utilizando estribos $^{(2)}$.

Según el tipo de discapacidad del paciente, se aplica la hipoterapia o la monta terapéutica. De- 
pendiendo de la evolución del paciente se puede cambiar de la hipoterapia a la monta terapéutica, lo cual otorga al paciente la oportunidad de practicar la equitación como futuro deporte.

\section{Principios Terapéuticos}

Los equinos permiten la base para la utilización de la equitación como terapia: transmiten su calor corporal, transmiten impulsos rítmicos, y, transmiten un patrón de locomoción equivalente al patrón fisiológico de la marcha humana.

El calor corporal del caballo es de $38^{\circ} \mathrm{C}$, convirtiéndose en un instrumento calorífico para distender y relajar musculatura y ligamentos, y estimular la sensopercepción táctil.

La relajación y elongación de los aductores provoca una liberación del cinturón pélvico, por lo que éste adquiere más flexibilidad y elasticidad, recuperando así su posición vertical correcta y su funcionalidad para la adaptación al movimiento del lomo del caballo. El asiento se hace cada vez más profundo y el calor que se trasmite desde el lomo del caballo penetra más en los músculos y ligamentos del cinturón pélvico. Se ha comprobado que el efecto distensor de los aductores se mantiene, incluso, hasta 6 horas después de una sesión terapéutica ${ }^{(2)}$.

En la segunda característica, el caballo trasmite por medio del movimiento de su lomo impulsos rítmicos al cinturón pélvico, a la columna vertebral y a los miembros inferiores del jinete. Al caminar en paso se trasmiten de 90 a 110 impulsos por minuto a la pelvis del jinete; al caminar en trote aumenta la cantidad y la intensidad de éstos. Los impulsos los provocan los músculos lumbares y ventrales del caballo, que se contraen y distienden alternadamente en forma rítmica y regular en el paso y el trote. Cuando los miembros posteriores del caballo se adelantan alternadamente debajo del centro de gravedad, se provoca una elevación alterna de la grupa y de la musculatura lumbar del caballo. Este movimiento hacia adelante fuerza al cinturón pél- vico del jinete a adaptarse con un movimiento basculante. Los impulsos fisiológicos se propagan hacia arriba por medio de la columna vertebral hasta la cabeza, provocando reacciones de equilibrio y enderezamiento del tronco.

La respuesta dinámica del jinete a los impulsos fisiológicos emitidos es un acto de coordinación fina del tronco y de la cabeza, por lo que es un excelente entrenamiento de la coordinación psicomotriz del tronco y de la cabeza sinutilizar las piernas; así se logra la estabilización dinámica de estas partes, lo que constituye la base para la ejecución de la marcha independiente. ${ }^{(2)}$

El tercer principio terapéutico, la transmisión de un patrón de locomoción tridimensional, adquiere especial importancia en la hipoterapia en el tratamiento de disfunciones neuromotoras.

El cerebro humano no sólo registra movimientos aislados, sino toda una gama de patrones motores. El patrón fisiológico de la marcha humana que el paciente realiza durante la monta se graba en el cerebro y con el tiempo se automatiza, lo que posibilita su transferencia a la marcha pedestre. ${ }^{(2)}$

No hay que olvidar que la terapia ecuestre se realiza en íntimo contacto con un animal.

El animal doméstico exhibe características muy positivas en su trato con el humano, como es la falta total de reacciones vengadas o rencorosas; el animal permite y busca el contacto corporal con el humano, entiende sin palabras. Se expresa con gestos claros y directos, nunca rechaza a quien se acerque amablemente y no conoce compasión, por lo cual tiene un comportamiento natural con cualquier persona ${ }^{(2)}$. Todas estas características importantes para el desarrollo del ser humano son elementos básicos en la terapia ecuestre, que utiliza a un animal como medio terapéutico.

El acompañamiento de un equipo multidisciplinario en los procesos terapéuticos permite observar la complejidad de pacientes con retraso 
psicomotor y el mejoramiento de la calidad de vida del entorno familiar, académico y social de los discapacitados.

Una consulta frecuente en neuropediatría es el retraso del desarrollo neurológico. La detección precoz permite un diagnóstico etiológico y tratamiento oportuno. En éste, se debe incluir un grupo multidisciplinario de profesionales de salud física y mental, educación y rehabilitación. Sólo es posible llevar a buen término el manejo de un paciente con déficit trabajando con un equipo articulado $^{(3)}$.

El retraso psicomotor en los niños está generalmente relacionado con lesiones o anomalías serias en el sistema nervioso central. Esta discapacidad hace referencia específicamente al retraso en el desarrollo de las destrezas cognitivas (como el lenguaje) y en el desarrollo de las destrezas motoras (como caminar).

Este trabajo tuvo por objetivo evaluar la contribución de la habilitación cognitiva asistida por la hipoterapia en el mejoramiento los aspectos cognitivos y psicomotores en paciente con retraso psicomotor en comparación con técnicas convencionales con igual objetivo.

\section{Metodología}

Para el desarrollo del proyecto de investigación se planteó el enfoque cuantitativo, este enfoque se basa en la medición numérica, el conteo y frecuentemente en el uso de las estadística para establecer con exactitud patrones de comportamiento en una población ${ }^{(4)}$. Por otra parte se utilizaron diseños cuasi-experimentales, éstos juegan un papel primordial en los contextos de investigación aplicada, el objetivo de estos diseños consiste en comprobar el efecto de determinados tratamientos terapéuticos o programas de intervención social o educativa(5).

Por último el Diseño $\mathrm{ABAB}$, es un plan de investigación cuasi-experimental en el que la secuencia es: línea base-tratamiento-retirada del tratamiento-tratamiento. Este se denomina vuelta atrás, debido a que después de una fase de tratamiento se vuelve a la situación anterior, es decir, una fase sin tratamiento. Como ventaja de la vuelta atrás es que con la retirada del tratamiento podemos poner de manifiesto la influencia de otras variables que han actuado simultáneamente.

\section{Paciente}

La paciente fue una infante de siete años nueve meses, de apariencia delgada, estatura acorde a su edad, perteneciente a una familia conformada por el padre, de 44 años, madre de 33 años y una hermana menor de tres años. La menor presenta dificultades en el aprendizaje, problemas de equilibrio y lenguaje poco articulado. A nivel emocional presenta cambios bruscos de humor sin razón aparente, es rebelde, nerviosa, malgeniada, tímida, inquieta y apegada. Es de notar que el embarazo fue programado y deseado, pero de alto riesgo, el parto fue inducido con utilización de fórceps, lactancia materna hasta la edad de tres años.

Para la recolección de la información se aplicó el cuestionario CUMANíN el cual se ha baremado con una muestra muy amplia de 879 sujetos procedentes de diversos centros; por otra parte, la escala McCarthy, la cual cuenta con una escala general cognitiva cuyo coeficiente de fiabilidad para el procedimiento de dos mitades para los grupos fue de 0.93 . Los promedios en las demás escalas varían de 0.79 a 0.88 . Los coeficientes de fiabilidad también reflejan un alto grado de estabilidad, con un valor aproximado de 0.90 en la escala GC y correlaciones que variaban de 0.75 y 0.89 en las demás escalas.

\section{Resultados}

Para realizar el análisis de los datos arrojados por el estudio es necesario aclarar que por ser un diseño $n=1$ se realizó un análisis descriptivo de los cambios en las puntuaciones de los instrumentos de medición. 
Al establecer la línea base del sujeto se puede evidenciar puntuaciones por debajo del centil 50 en las dimensiones principales que evalúa la prueba CUMANIN, exceptuando el lenguaje articulatorio, visopercepción y memoria icóni$\mathrm{ca}$, mientras que en las dimensiones auxiliares obtuvo puntuaciones superiores al centil 50 en lectura, escritura (Figura 1).

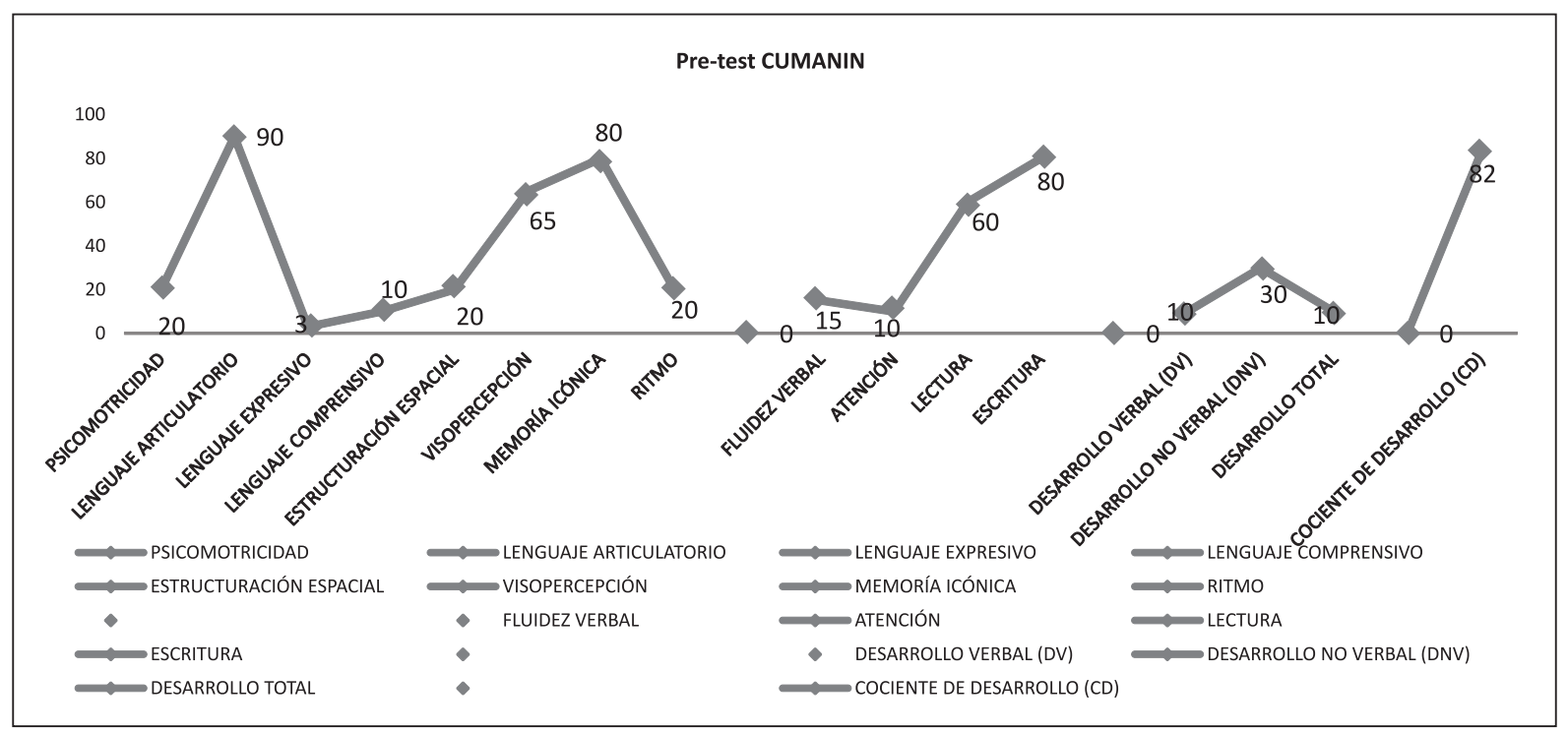

Figura 1. Pretest prueba CUMANIN

Al revisar las puntuaciones del sujeto en la prueba se evidencianpuntuaciones por debajo de la media (50 \pm 10$)$, presentando su mejor desempeño en lo perceptivo-manipulativo, con relación a la general cognitiva (media 100 \pm 16 ), el sujeto puntúa muy por debajo del valor medio (Figura 2).

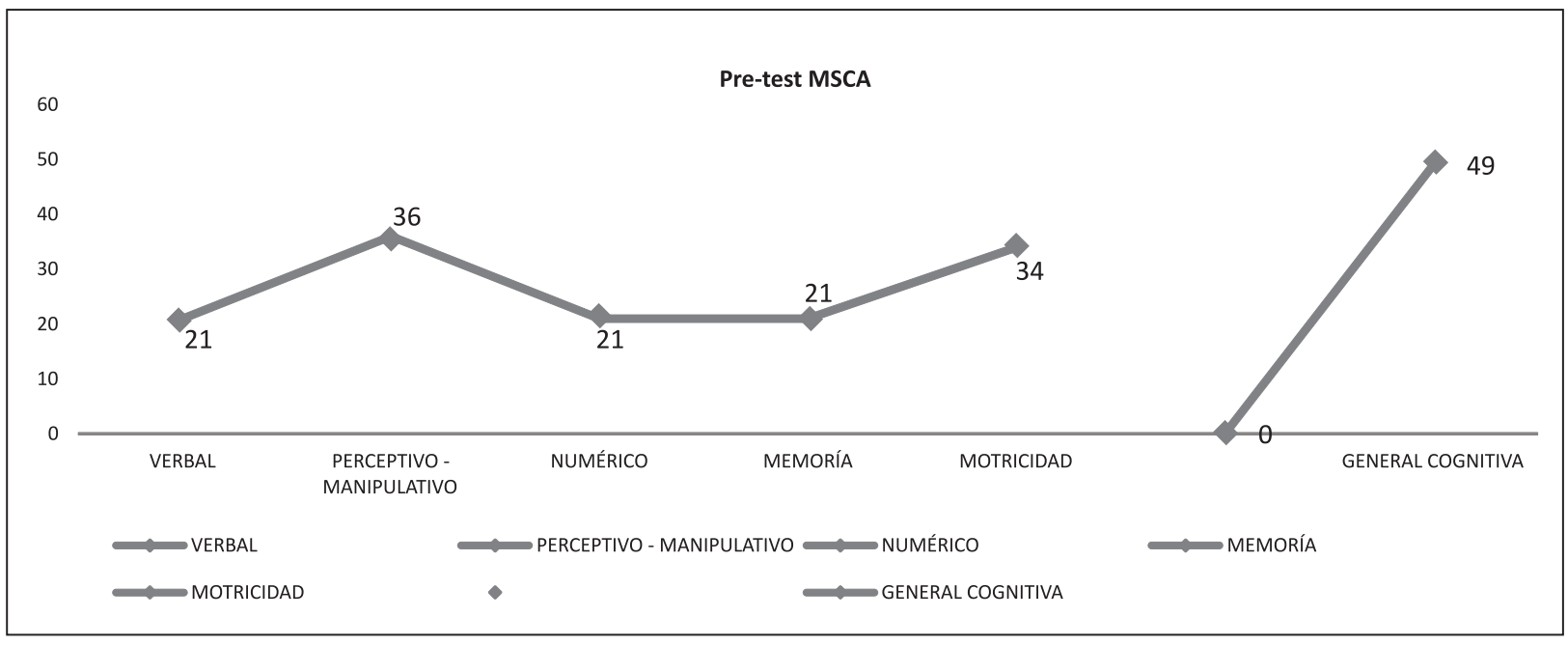

Figura 2. Pretest prueba MSCA 
Al realizar la segunda medición, los resultados evidenciaron puntuaciones superiores al centil 50 en las dimensiones lenguaje articulatorio, lenguaje comprensivo y ritmo,percepción, y memoria icónica; dentro de las dimensiones auxiliares la lectura, escritura presentaron puntuaciones superiores al centil 50 (Figura 3)

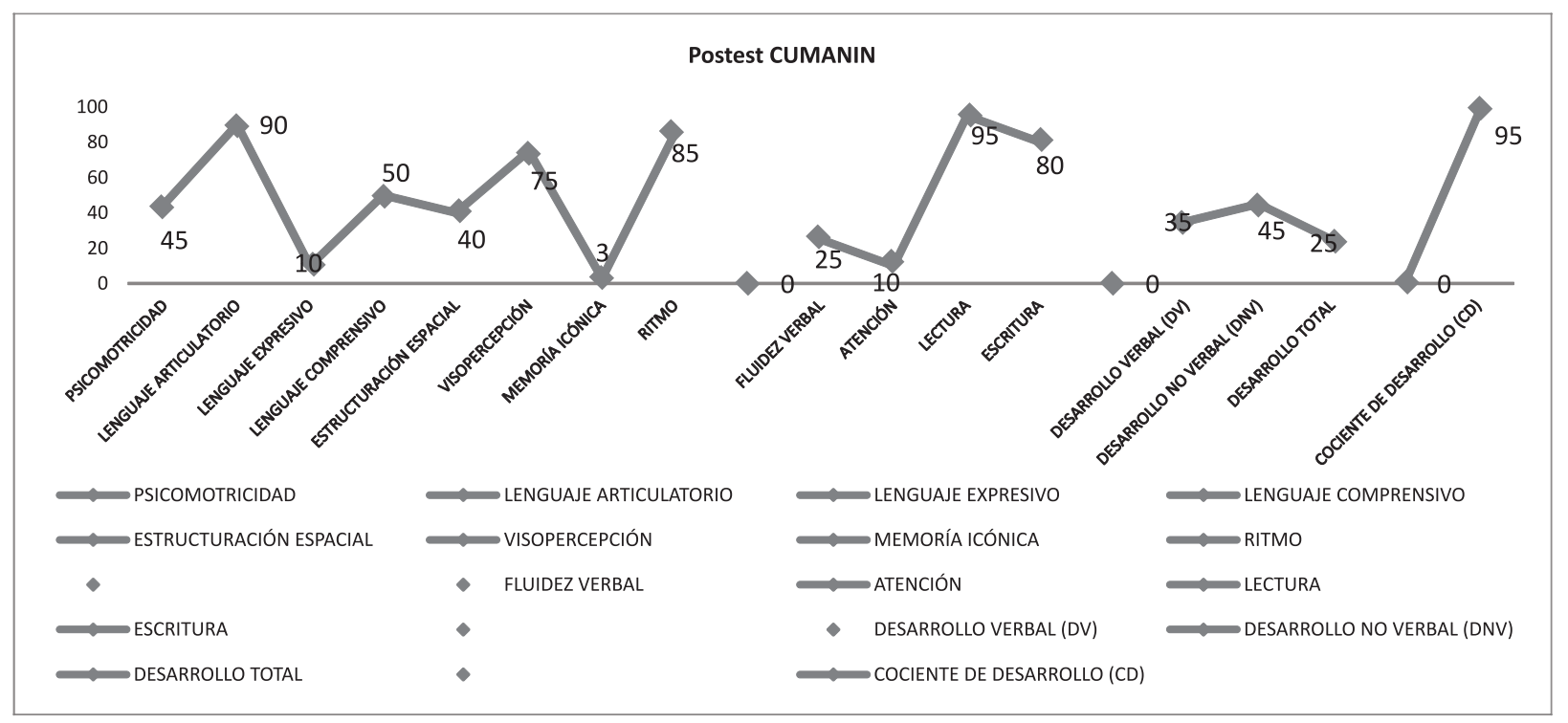

Figura 3. Postest prueba CUMANIN

Las puntuaciones del sujeto en la pos prueba evidencia su mejor desempeño en la escala verbal y motricidad (puntuaciones inferiores a la media), la general cognitiva (media $100 \pm 16$ ), el sujeto puntuó 66 muy por debajo del valor medio (Figura 4).

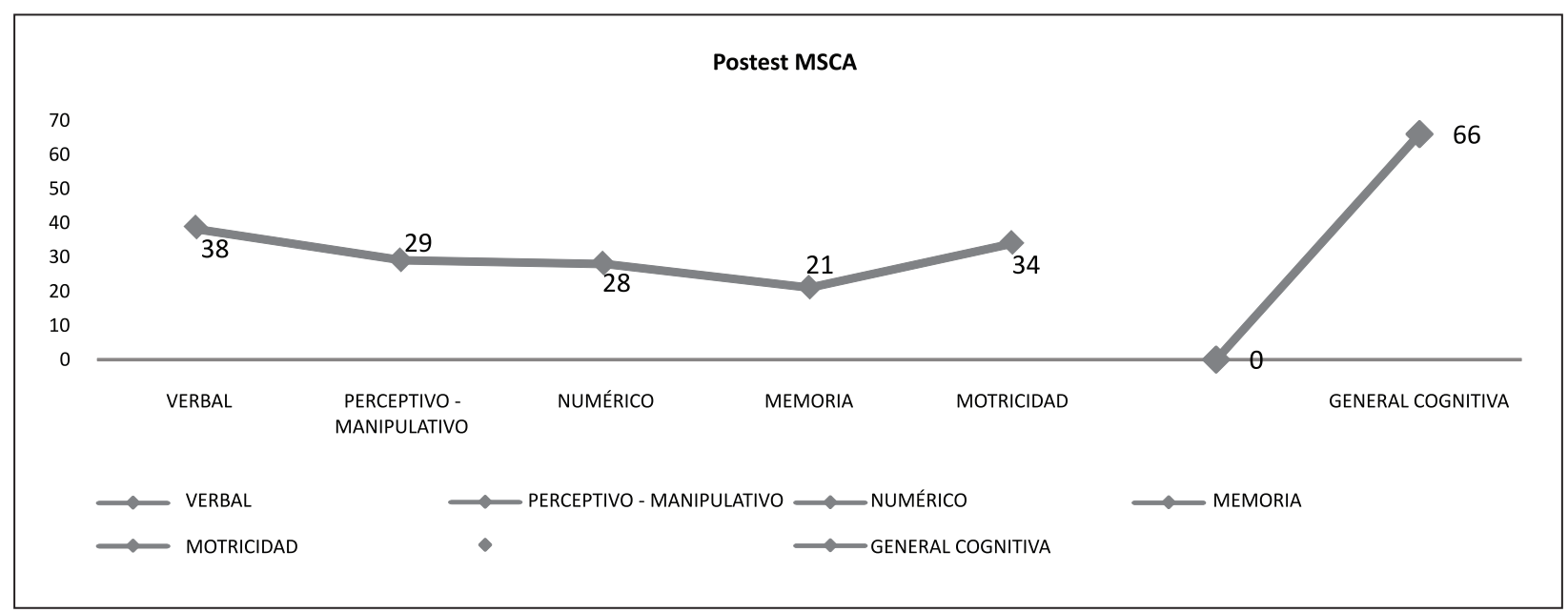

Figura 4. Pretest prueba MSCA 
Con relación a las puntuaciones obtenidas por el sujeto en el CUMANIN se puede establecer que se presentaron cambios positivos en psicomotricidad, lenguaje comprensivo, estructu- ración espacial, rítmico-temporal, lectura. De igual forma, se presentó descenso en la escala de memoria icónica (Figura 5).

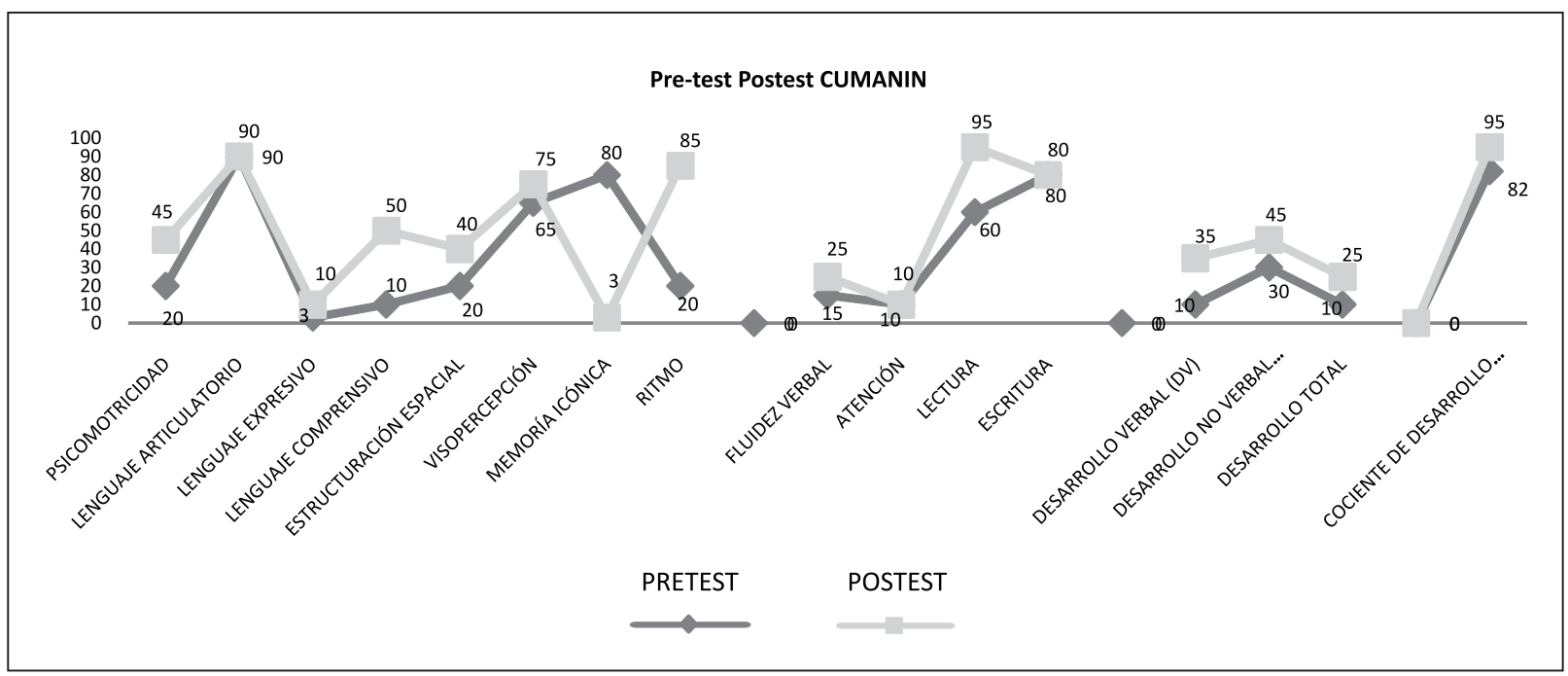

Figura 5. Pre test y Pos test prueba CUMANIN luego de la intervención

Por su parte, el MSCA que evalúa en carácter lúdico, los aspectos cognitivos y psicomotores se evidenciaron cambios positivos en la esca- la verbal y numérica, y un decremento a nivel perceptivo-manipulativo, sin embargo a nivel general se evidenciaron cambios luego de la intervención (Figura 6).

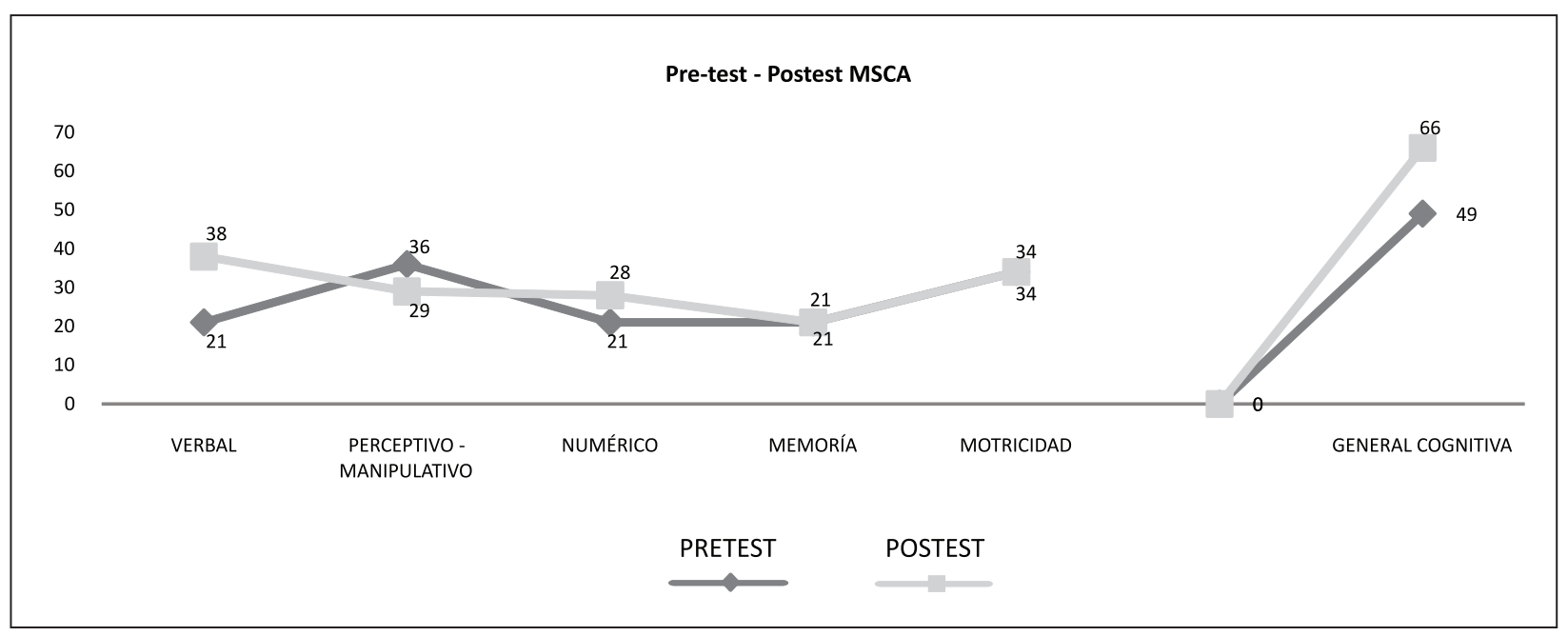

Figura 6. Pretest-postest prueba MSCA luego de la intervención 
A continuación se presentan los puntos fuertes obtenidos por el sujeto en la prueba (Tabla 1)

Tabla 1. Puntos fuertes luego de la intervención

\begin{tabular}{|c|c|c|c|}
\hline \multicolumn{4}{|c|}{ Puntos fuertes } \\
\hline No. & Test & Pretest & Postest \\
\hline 3 & Memoria pictórica & & $81 / 2$ años \\
\hline 12 & Copia de dibujos & $81 / 2$ años & $71 / 2$ años \\
\hline 13 & Dibujo de un niño & $61 / 2$ años & $71 / 2$ años \\
\hline 14 & Memoria numérica (II) & $61 / 2$ años & \\
\hline 17 & Opuestos & & $71 / 2$ años \\
\hline
\end{tabular}

\section{Discusión}

Luego de los hallazgos encontrados se ha podido determinar que el estado de funcionamiento de los procesos de psicomotricidad, lenguaje, atención, estructuración espacial, visopercepción, memoria, estructuración rítmico-temporal, lateralidad, perceptivo-manipulativa, cuantitativa, memoria y motricidad en el sujeto de estudio; antes, durante y después de la terapia presentaron cambios como consecuencia de la aplicación de la hipoterapia, lo cual determina para este caso un indiciofavorable en las áreas cognitivas y psicomotoras, y se ha podido evidenciar la recuperación de los aspectos cognitivos y psicomotores del desarrollo de la niña en cuestión; este proceso se relaciona con la investigación $^{(6)}$ quienes refieren los significativos beneficios a nivel estadístico en la habilidad motora de niños con retraso motor mediante siete semanas de tratamiento con terapia ecuestre. De igual forma durante el transcurso de la investigación se planteó el tratamiento en 10 semanas con asistencia de dos veces por semana a la fundación.

Otros autores plantean que la maduración que logra a nivel psiconeuromotriz, sensitivo, y social permite constatar que la terapia ha sido eficaz, además, van a existir ejes que se desarrollen más que otros en función del trastorno, personalidad y entorno del niño(7), con relación a lo anterior, es necesario mencionar que los cambios presentados en el sujeto deben ser atribuidos no solo al proceso de intervención, sino a la interacción con su entorno, siendo este eje fundamental en los procesos evolutivos y de desarrollo de los individuos. Lo anterior se asocia a las mejoras en las habilidades motoras, habilidades del lenguaje, en el control emocional, apertura social, relaciones de pares, y auto concepto, que posibilita las habilidades para el trabajo y la autoconfianza.

Destaca cómo el efecto terapéutico no se limita solo a la influencia del movimiento sobre los ajustes posturales del niño, sino que la actividad por su naturaleza se torna agradable y estimulante, y una oportunidad de mejorar los procesos cognoscitivos, el lenguaje y la socialización, estos asociados al desarrollo terapéutico propuesto para el estudio. ${ }^{(8)}$

De igual forma, se coincide con las consideraciones teóricas de la mayoría de autores citados en la revisión bibliográfica que denotan la eficacia de la técnica hipoterapéutica, ya que se pudo evidenciar en los logros obtenidos en la niña que fue objeto de la presente investigación.

Una de las principales limitaciones de la investigación está asociada a los instrumentos utilizados, ya que estos no están normalizados para la población colombiana, por consiguiente, las mediciones se realizaron con estos baremos. De igual forma la dificultad para encontrar los sujetos con el diagnóstico de retraso psicomotor y que cumplieran los criterios de inclusión (para aplicación de las pruebas psicológicas), y que ésta no estuviera asociada a otras patologías que pudieran afectar el desarrollo de la intervención, retrasó el desarrollo del estudio.

\section{Conclusiones}

Este estudio aporta evidencia teórica y empírica de cómo evoluciona un niño con trastorno psicomotriz por medio de la terapia ecuestre.

La hipoterapia es un tratamiento coadyuvante a otros tratamientos, y debe considerarse como 
parte de un conjunto de acciones terapéuticas dirigidas a la rehabilitación de la discapacidad, aumentando el desarrollo de los potenciales residuales y generando nuevas capacidades.

Al ser una actividad realizada al aire libre, en contacto directo con la naturaleza, en espacio abierto, tiene un efecto favorable en la esfera psicológica y emocional del paciente, que con frecuencia, a causa de la discapacidad, se ve limitado a realizar actividades de este tipo.

Se puede concluir que la aplicación del procedimiento hipoterapéutico (variable independiente) mejoró el índice de desarrollo del sujeto a nivel de psicomotricidad, lenguaje, estructuración espacial y visopercepción.

Las áreas que se mantuvieron estables fueron lenguaje articulatorio, atención y escritura

Se presentó un decremento en la memoria icónica, (el sujeto tiende a memorizar los objetos sencillos) lo que conlleva al registro de la memoria sensorial relacionada con el dominio visual.

\section{Declaración sobre conflicto de intereses}

Los autores declaran que no poseen conflictos de intereses.

\section{Recomendaciones}

Se recomienda fomentar el desarrollo de procesos investigativos tendientes a demostrar la incidencia de la hipoterapia en el mejoramiento de la calidad de vida de los usuarios.

Validar y normalizar pruebas para la región encaminadas a medir los diferentes cambios que se pueden presentar luego de un proceso de intervención hipoterapéutico y así poder establecer baremos propios para tal medición.

Dar a conocer a la comunidad el procedimiento hipoterapéutico desarrollado para la intervención del retraso psicomotor y así establecer los cambios que se pueden presentar a raíz de este.

\section{Bibliografía}

1. DEL ROSARIO-MONTEJO, O., et al., Effectiveness of equine therapy in children with psychomotor impairment. Neurologia, 2014. 20(14): p. 00020-6.

2. NASCHERT, E.G., Equinoterapial Equinotherapy: La Rehabilitacion Por Medio Del Caballo. 2006: Editorial Trillas Sa De Cv.

3. SCHLACK, L. Retraso del desarrollo psicomotor. Manual de Pediatria http://escuela.med.puc.cl/ paginas/publicaciones/manualped/RetrDessPs. html.

4. SALGADO LÉVANO, A.C., Investigación cualitativa: diseños, evaluación del rigor metodológico y retos. Liberabit, 2007. 13: p. 71-78.

5. COOLICAN, H., Introduction to Research Methods in Psychology, ed. H. Arnold. 2006, London.

6. WINCHESTER, P., et al., The effect of therapeutic horseback riding on gross motor function and gait speed in children who are developmentally delayed. Phys Occup Ther Pediatr, 2002. 22(3-4): p. 37-50.

7. CARDO, M., El niño y el caballo desde una perspectiva psicológica. Psicología y Psicopedagogía, 2011. 8(21).

8. MereGillano, G., Hippotherapy. Phys Med Rehabil Clin N Am, 2004. 15(4): p. 843-54. 